\title{
Theoretical study of the porosity effects on the shock response of graphitic materials
}

\author{
Nicolas Pineau ${ }^{1, a}$, Emeric Bourasseau ${ }^{1}$, Jean-Bernard Maillet ${ }^{1}$, Laurent Soulard ${ }^{1}$, and David Hébert ${ }^{2}$ \\ ${ }^{1}$ CEA/DAM/DIF, 91297 Arpajon, France \\ ${ }^{2}$ CEA/DAM/CESTA, 33116 Le Barp, France
}

\begin{abstract}
In this paper we present a theoretical study of the shock compression of porous graphite by means of combined Monte Carlo and molecular dynamics simulations using the LCBOPII potential. The results show that the Hugoniostat methods can be used with "pole" properties calculated from porous models to reproduce the experimental Hugoniot of pure graphite and diamond with good accuracy. The computed shock temperatures show a sharp increase for weak shocks which we analyze as the heating associated with the closure of the initial porosity. After this initial phase, the temperature increases with shock intensity at a rate comparable to monocrystalline graphite and diamond. These simulations data can be exploited in view to build a full equation of state for use in hydrodynamic simulations.
\end{abstract}

\section{Introduction}

The responses of carbon materials under high pressure and temperature constraints have motivated a large number of experimental and theoretical studies in the academic and applied physics fields [1-9]. However building accurate equations of state (EOS) remains a very challenging problem due to a lack of reliable experimental data on the thermal effects associated to its diverse forms and transformations: indeed temperature measurements can be particularly difficult under extreme, highly dynamic constraints. This difficulty increases in the case of porous systems due to the differences between the initial samples used in the reference experiments. For instance, experimentally shock-compressed graphite can span porosity rates ranging up to more than $20 \%$, yielding graphite to diamond (G/D) transition pressures from 18 to $45 \mathrm{GPa}$. These values lie substantially above the estimated G/D coexistence line (in the 5-8 GPa range according to experiments and simulations $[2,10,11])$, which signs a possible metastability of shocked graphite in the diamondpredominant regime (reversely, diamond is well-known to be metastable in the low temperature/pressure regime, where graphite is the most stable phase). This effect results in a wide spread of experimental data in the G/D transition regime [12-17].

Theoretical studies of the shock compression of graphite are scarce. Recently, a DFT-based molecular dynamics study yielded one point of the Hugoniot curve beyond the G/D transition using the Multi-Scale Shock Technique [18] (MSST-DFT). Then the full Hugoniot curve of monocrystalline graphite was calculated [9] using the LCBOPII empirical potential $[19,20]$, in excellent agreement with MSST-DFT, but with a large overestimation of the G/D transition pressure (G/D).

\footnotetext{
${ }^{a}$ Corresponding author: nicolas.pineau@cea.fr
}

In this paper we address the shock compression of porous graphite using atomistic (Monte Carlo and molecular dynamics) simulations with the LCBOPII potential using specifically designed porosity models. We compare our Hugoniot data to experimental results and discuss the role of the sample porosity on the resulting equation of state.

\section{Numerical setup}

This study is based on a combination of Monte Carlo (MC) and molecular dynamics (MD) simulations. We ran the MC simulations with the GIBBS code [21] and the MD simulations with the STAMP code [22]. The MC simulations used samples of limited sizes (up to 5.000 atoms) of bulk graphite and diamond with 3D periodic conditions. The MC Hugoniot curves were calculated using the SCA method [23,24]. We used MD to calculate the specific energies of the various porosity models at $300 \mathrm{~K}$, in the NVT ensemble.

We designed two porosity models for the porous Hugoniot calculations: a so-called "mosaic" model to treat the intergranular component of the porosity, and a socalled "intragranular" model to treat the porosity inside graphite grains. We chose a pole density of $1.81 \mathrm{~g} / \mathrm{cm}^{3}$ corresponding to a $20 \%$ porosity: this density is close to the densities of the experimental samples presented for comparison. In the mosaic model (Fig. 1-left) the system consists of a $2 \times 2 \times 2$ stack of graphite cubes of size $6.1 \times 6.0 \times 6.0 \mathrm{~nm}^{3}$ with alternating orientations: the spacing between the cubes is adjusted to obtain the desired density. In the intragranular model (Fig. 1-right) a paralleliped cavity of size $7.8 \times 7.5 \times 7.6 \mathrm{~nm}^{3}$ is cut into a bulk graphite cube of size $13.6 \times 13.2 \times 13.3 \mathrm{~nm}^{3}$ : the fraction of removed atoms is adjusted to reach a density within $0.1 \%$ of the mosaic model. These two models may be considered as characteristic of the different types of 


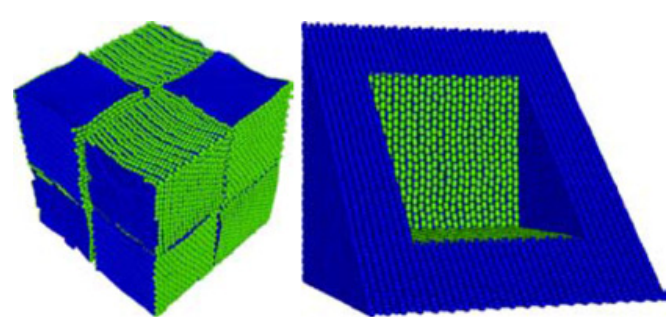

Figure 1. Structure of the mosaic (left), sc-intragranular (right). In order to highlight the geometry of the pores, we colored the atoms according to their coordination: blue for the fully coordinated graphite atoms $\left(\mathrm{sp}^{2}\right)$, green for under-coordinated atoms.

porosity that can be found in a compacted powder (inside a grain for the intragranular case and between grains for the mosaic case); however the defect sizes probably lie at the lower boundary of the actual experimental ranges, thus maximizing the "porous energy" with respect to real samples. We obtained specific energies of $-56.9 \mathrm{MJ} / \mathrm{kg}$ and $-57.8 \mathrm{MJ} / \mathrm{kg}$ for the mosaic and intragranular case (to be compared to a specific energy of $-58.6 \mathrm{MJ} / \mathrm{kg}$ for bulk graphite at $300 \mathrm{~K})$.

\section{Results and discussion}

In Fig. 2 we present the results of the MC hugoniostat (MC-SCA) calculations for bulk and porous graphite and diamond with a comparison to a set of experimental data for graphite powders compacted to $1.76 \mathrm{~g} / \mathrm{cm}^{3}$ and $1.88 \mathrm{~g} / \mathrm{cm}^{3}$.

The results show that the Hugoniot of bulk graphite is close to the experimental data for pressures lower than $20 \mathrm{GPa}$, whereas the diamond shock pressure is substantially underestimated on the full range. These discrepancies are corrected by the use of the properties of porous graphite at the "pole": in particular the increment in shock pressure is sufficient to fill the gap between bulk diamond and experiments, yielding an excellent agreement for pressures superior to $40 \mathrm{GPa}$. This effect is also visible, although less marked, for porous graphite.

For the temperature EOS, the agreement between the SESAME data and our calculations for bulk graphite is excellent. However the impact of porosity on the shock temperature is substantial and not accounted for in the EDM3 porous EOS. In particular, a sharp temperature increase is found for low shock intensities $(+700 \mathrm{~K}$ for the intragranular case, $+1200 \mathrm{~K}$ for the mosaic case) which may result from the closure of the porosity induced by the shock: similar trends are found starting from graphite or diamond. After this initial heating phase, the shock temperatures increase at distinct, quasi constant rates for graphite and diamond ( $37.4 \mathrm{~K} / \mathrm{GPa}$ vs. $\sim 63.3 \mathrm{~K} / \mathrm{GPa}$ respectively) reflecting the difference in heat capacity between the two bulk solids $(0.71 \mathrm{~kJ} / \mathrm{kg} / \mathrm{K}$ [26] vs. $0.52 \mathrm{~kJ} / \mathrm{kg} / \mathrm{K}$ [27] at $300 \mathrm{~K})$. Note that the change in magnitude of the computed slopes agree qualitatively with the ones estimated by the bulk EOS $(\sim 12.7 \mathrm{~K} / \mathrm{GPa}$ vs. $\sim 42.8 \mathrm{~K} / \mathrm{GPa}$ ).

The hugoniostat simulations of graphite and diamond show strong metastabilities of the bulk phases (at low
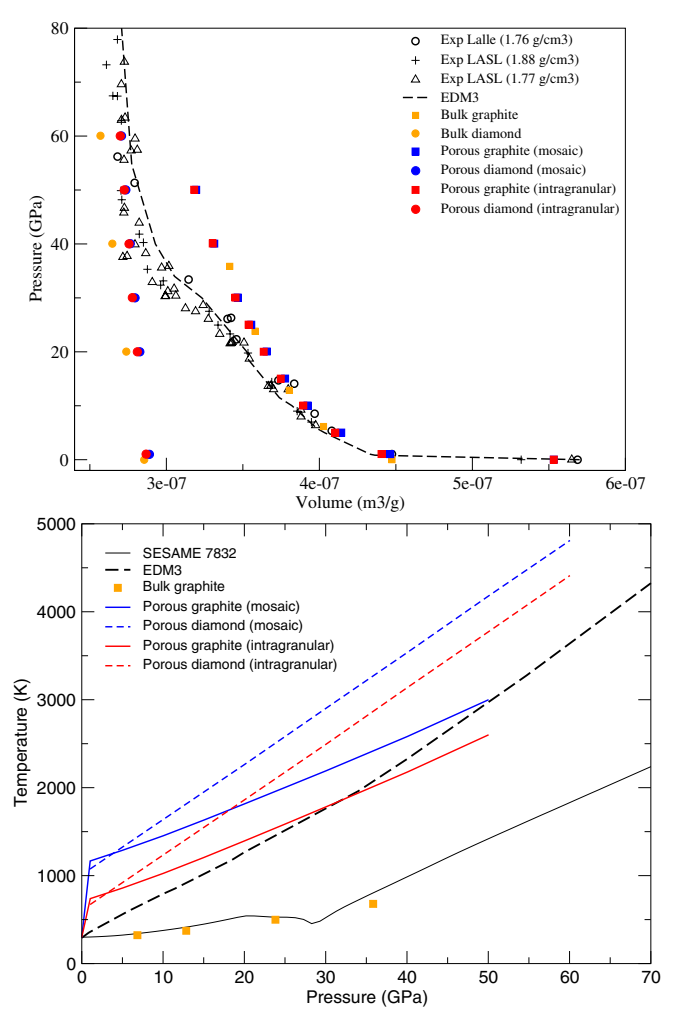

Figure 2. $P(V)$ and $T(P)$ Hugoniot diagrams of the bulk and porous systems calculated from MC simulations. The orange symbols are for the bulk graphite pole, blue lines for the mosaic porous pole, red lines for the intragranular porous pole. Full lines are for graphite simulations and dashed lines for diamond. The black symbols are a few set of experimental measurements (the corresponding pole densities are given in the legend). The corresponding SESAME (bulk graphite, $T(P)$ diagram) and EDM3 [25] (porous carbon, both diagrams) equations of state are provided (full and dashed black lines respectively).

and high pressure for diamond and graphite respectively). Accordingly these simulations fail to reproduce the intermediate states found experimentally between 20 and $40 \mathrm{GPa}$, which likely correspond to the transition of graphite toward diamond. This result is not fully surprising since i) our samples have limited sizes which may hinder the nucleation of diamond in shocked graphite (and reversely), and ii) graphite and diamond are known to be strongly metastable beyond their respective thermodynamic domains. This draws a limit to the validity of the calculated shock temperatures in view to build a full EOS for shocked carbon, and calls for a new strategy in order to explore this intermediate regime. Note that the large dispersion of the experimental data in the 20-40 GPa range could result from the partial shock-conversion of the initial graphite to diamond, with a probable influence of the initial sample density and structural properties on the $\mathrm{G} / \mathrm{D}$ transition rate.

The results at low and high pressure can be exploited in view to construct a partial EOS for shocked porous graphite, including the temperature which is very difficult to access experimentally. First a domain of confidence must be drawn for the pure graphite and diamond phases. The large number of available shock experiments tells 


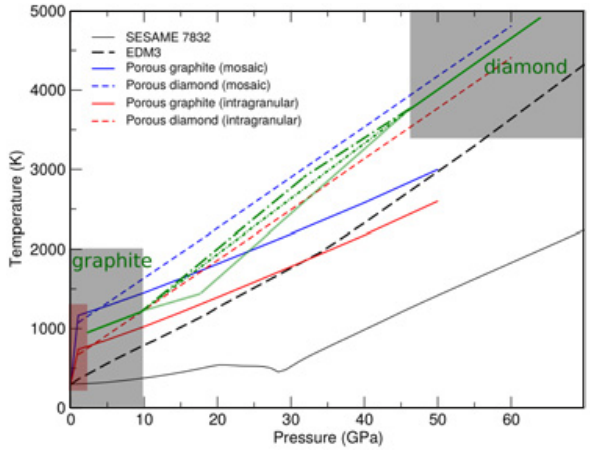

Figure 3. Same as Fig. 2: domains of confidence for graphite and diamond (grey regions) and schematics of potential EOS models are proposed (green lines).

us that graphite is always preserved for shock pressures lower than $15 \mathrm{GPa}$, and diamond is always found above $40 \mathrm{GPa}$. Therefore we will rely on the LCBOPII Hugoniot for pure graphite and pure diamond in these two respective low and high pressure domains, either in the $P(V)$ or the $T(P)$ diagrams. Examples of potential equations of state in the $T(P)$ diagram are given in Fig. 3. Note that new experimental measurements of the shock temperature appear necessary to validate the final EOS, in particular in the low shock intensity regime, where the sharp temperature increase is predicted.

\section{Conclusion}

We used MC and MD simulations to unravel the behaviour and EOS of polycrystalline graphite under shock compression. In the MC-SCA framework, the complex structure of the polycrystalline samples was accounted for by using a pole energy estimated from several model of porous structures, corresponding the variety of porosity types in granular materials.

Our results show that a reasonable quantitative estimation of the porous energy yields shock compression results in good agreement with the available $P(V)$ experimental data in the $\mathrm{P}<15 \mathrm{GPa}$ and $\mathrm{P}>40 \mathrm{GPa}$ regimes. The simulations predict a sharp heating in the low compression regime which may be assigned to the closure of the porosity. These data can be used to build an EOS with reasonable confidence in the low compression and high compression regimes, where respectively graphite and diamond are experimentally, and unambiguously, known to be the only stable form of shocked carbon.

Due to the strong metastability of graphite and diamond beyond their respective domains of predominance, the intermediate regime cannot be investigated using bulk graphite or diamond as the initial material. We are currently working on a new procedure using hybrid G/D samples in the MC-SCA framework: we believe that the use of pre-nucleated diamond in shocked graphite is a necessary step to explore this regime with small-scale atomistic simulations.

Besides, we use large-scale MD simulations to unravel the shock-compression mechanisms at play in porous carbon: in particular we investigate the dependance of the
G/D transition threshold on porosity, which may explain partially the major discrepancy between experimental and simulation Hugoniot curves.

This work shows that a set of simple but well designed atomistic simulations can be used to obtain reliable information on the thermodynamic response of complex materials. From these data, complete EOS can be proposed and used for finite element methods, in a multiscale approach. The key ingredients to a full model are an accurate potential energy function, a set of approximate structural models which, taken altogether, are representative of the complexity of the system, and experimental data in the full targeted thermodynamic range.

Further developments, or generalization, of temperature measurement setups applied to the shock compression of solids is indispensable in order to validate the thermal component of the proposed EOS.

\section{References}

[1] F.P. Bundy, J. Chem. Phys. 38, 618 (1963)

[2] F.P. Bundy, J. Chem. Phys. 38, 631 (1963)

[3] S.V. Zybin, M.L. Elert, C.T. White, Phys. Rev. B 66, 220102 (2002)

[4] H. Nagao, K.G. Nakamura, K. Kondo, N. Ozaki, K. Takamatsu, T. Ono, T. Shiota, D. Ichinose, K.A. Tanaka, K. Wakabayashi et al., Phys. Plasmas 13, 052705 (2006)

[5] J.H. Eggert, D.G. Hicks, P.M. Celliers, D.K. Bradley, R.S. McWilliams, R. Jeanloz, J.E. Miller, T.R. Boehly, G.W. Collins, Nature Physics 6, 40 (2010)

[6] R.S. McWilliams, J.H. Eggert, D.G. Hicks, D.K. Bradley, P.M. Celliers, D.K. Spaulding, T.R. Boehly, G.W. Collins, R. Jeanloz, Phys. Rev. B 81, 014111 (2010)

[7] R.Z. Khaliullin, H. Eshet, T.D. Kuhne, J. Behler, M. Parrinello, Nature Materials 10, 693 (2011)

[8] N.A. Marks, M. Lattemann, D.R. McKenzie, Phys. Rev. Lett. 108, 075503 (2012)

[9] N. Pineau, J. Phys. Chem. C 117, 12778 (2013)

[10] R. Berman, F. Simon, Z. Elektrochem. 59, 333 (1955)

[11] L.M. Ghiringhelli, J.H. Los, E.J. Meijer, A. Fasolino, D. Frenkel, Phys. Rev. Lett. 94, 145701 (2005)

[12] B.J. Alder, R.H. Christian, Phys. Rev. Lett. 7, 367 (1961)

[13] W.H. Gust, Phys. Rev. B 22, 4744 (1980)

[14] D.J. Erskine, W.J. Nellis, Nature 349, 317 (1991)

[15] W.J. Nellis, A.C. Mitchell, A.K. McMahan, J. Appl. Phys. 90, 696 (2001)

[16] K. Yamada, Y. Tanabe, Carbon 40, 261 (2002)

[17] G.I. Kanel', G.S. Bezruchko, A.S. Savinykh, S.V. Razorenov, V.V. Milyavskii, K.V. Khishchenko, High Temperature 48, 806 (2010)

[18] C.J. Mundy, A. Curioni, N. Goldman, I.F.W. Kuo, E.J. Reed, L.E. Fried, M. Ianuzzi, J. Chem. Phys. 128, 184701 (2008)

[19] J.H. Los, L.M. Ghiringhelli, E.J. Meijer, A. Fasolino, Phys. Rev. B 72, 214102 (2005) 
[20] J.H. Los, L.M. Ghiringhelli, E.J. Meijer, A. Fasolino, Phys. Rev. B 73, 229901(E) (2006)

[21] P. Ungerer, B. Tavitian, A. Boutin, Applications of molecular simulation in the oil and gas industry (IFP Publications, Paris, France, 2005)

[22] STAMP is a massively parallel MD code developped at CEA/DAM

[23] J.B. Maillet, G. Stoltz, Applied Math. Research eXpress 2008, abn004 (2009)
[24] E. Bourasseau, J.B. Maillet, N. Desbiens, G. Stoltz, J. Phys. Chem. A 115, 10729 (2011)

[25] D. Hébert, G. Seisson, I. Bertron, J. Chevalier, C. Thessieux, J. Quessada, S. Tastet, J. Phys. Conf. Ser. 500, 112057 (2014)

[26] S. Picard, D. Burns, P. Roger, Metrologia 44, 294 (2007)

[27] A.C. Victor, J. Chem. Phys. 36, 1903 (1962) 